\title{
La orientación al mercado, el dinamismo del entorno y la tipología estratégica de la pequeña y mediana industria: un análisis mediante modelo de ecuaciones estructurales
}

\section{The orientation to the market, the dynamism of the environment and the strategic typology of the small and medium industry: an analysis by means of model of structural equations}

Jaime Gustavo Gallo Mendoza

Universidad Internacional del Ecuador, Ecuador

Autor para correspondencia: ggallo@uide.edu.ec

Fecha de recepción: 31 de Julio de 2017 - Fecha de aceptación: 10 de Agosto de 2017

Resumen: En este trabajo se explica la relación de la orientación al mercado, el dinamismo del entorno competitivo y su estrategia; exponiendo de esta manera su influencia en los resultados empresariales en las pequeñas y medianas empresas ecuatorianas. El constructo "orientación al mercado - resultados organizacionales" tiene una alta influencia en los resultados empresariales, pero una baja influencia cuando interfiere la variable "dinamismo del entorno", debido a que la PyME ecuatoriana posee un bajo grado de orientación al marketing y las estrategias que han definido este tipo de empresas no son las adecuadas y les impide ser eficientes. La metodología está basada mediante el modelo de ecuaciones estructurales para el análisis del constructo. La tipología estratégica está basada en el modelo de "auto clasificación a través del método del párrafo de Miles y Snow (1978)". Se confirma que sí existe un vínculo entre la orientación al mercado, el dinamismo del entorno y su estrategia definida, lo cual se traduce en resultados organizacionales efectivos, que, si bien no alcanzan niveles significativos en el desarrollo de las Pyme ecuatorianas, no debe sustentarse dicho resultado en falta o deficiencia de creatividad e iniciativa personal, sino en la actual cultura empresarial existente.

Palabras clave: orientación al mercado; dinamismo del entorno; estrategia

Abstract: This paper explains the relationship of market orientation, the dynamism of the competitive environment and its strategy; exposing its influence on business results in small and medium-sized Ecuadorian companies. The "market orientation - organizational results" construct has a high influence on business results, but a low influence when the variable "dynamism of the environment" interferes, because the Ecuadorian SME has a low degree of marketing orientation and the strategies that have defined this type of companies are not appropriate and prevents them from being efficient. The methodology is based on the structural equations modeling for construct analysis. The strategic typology is based on the model of "self-classification through the method of the paragraph of Miles and Snow (1978)". It is confirmed that there is a link between the market orientation, the dynamism of the environment and its defined strategy, which translates into effective organizational results, which, although they do not reach significant levels in the development of Ecuadorian SMEs, should not be sustained this result in lack of creativity and personal initiative, but in the current existing business culture.

Key words: market orientation; dynamism of the environment; strategy 


\section{Introducción}

El modelo de ecuaciones estructurales o SEM (Structural Equation Model) es una técnica de análisis multi-variante para probar y estimar las relaciones causales, a partir de datos estadísticos y asunciones cualitativas. Este análisis considera un estudio empírico sobre las pequeñas y medianas industrias ecuatorianas de un sector productivo en particular, para conocer su grado de orientación al mercado y su impacto en los resultados empresariales.

El estudio demuestra que existe una relación positiva, pero cuando interviene una variable de control, su impacto es mayor.

\section{Los modelos de ecuaciones estructurales}

Estos modelos permiten construir esquemas explicativos, es decir, teorías que viabilicen una comprensión de la realidad y de los fenómenos observados (Ruiz, Pardo y San Martín, 2010). Son una familia de modelos estadísticos multivariados que permiten estimar el efecto y las relaciones entre las variables. Examinan simultáneamente una serie de relaciones de dependencia y analizan cuando una variable dependiente se convierte en independiente en otras relaciones.

\section{Causalidad}

El potencial de este modelo permite representar el efecto causal entre sus variables. También se puede estimar el parámetro correspondiente a ese efecto, pero se debe tener claro que su valoración no demuestra la existencia de causalidad. La existencia de una relación causal entre las variables debe sustentarse en la articulación teórica del modelo y no en la estimación de los datos (Ruiz, Pardo y Martín, 2010).

Estos modelos no prueban la causalidad, solamente permiten seleccionar hipótesis causales relevantes, eliminando aquellas no sustentadas por la evidencia empírica. Se especifican una serie de relaciones y luego se evalúan cuáles se representan en los datos recolectados empíricamente (Weston y Gore, 2006).

\section{Modelo teórico}

\section{La orientación al mercado}

(Narver, Slater, 1990; (Kohli, Jaworski, 1990) consideran a la orientación al mercado u orientación al marketing con un enfoque centrado en el consumidor y en los competidores; por lo que se ve más como una filosofía con orientación externa y que afecta a toda la organización, de forma que debe asumirse por toda la organización y no sólo por el departamento de marketing. También se diferencia de la orientación a las ventas, ya que, en este caso, la empresa consigue su objetivo cuando coloca el producto; mientras que la orientación al mercado no sólo es la venta, ya que existen más tareas como la de asegurar la repetición de compra, analizar la satisfacción del cliente, u ofrecer un servicio postventa (Levitt, 1983). 
Llonch y Waliño (1996) han definido la orientación al mercado como un rasgo de la cultura organizativa que nace de la aplicación del concepto de marketing en la empresa. Entre las características que destacan en este tipo de organización cabe señalar el que se trata de una gestión orientada al entorno, en la que se enfatiza en los procesos de marketing, los cuales serán puestos en práctica por toda la empresa y en que las responsabilidades recaen en el director general.

En el presente estudio sobre la orientación conviene señalar que las definiciones más influyentes han sido la Kohli con Jaworski y la de Narver con Slater.

Shapiro (1988) ha señalado que una empresa está orientada al mercado si: la información sobre el mercado se distribuye por todas las áreas de la empresa; todas las áreas funcionales participan en la toma de decisiones estratégicas y tácticas de manera coordinada y se ejecutan con un sentido de compromiso.

Webster (1988) ha propuesto que una empresa está orientada al mercado si cumple los siguientes requisitos: los valores y creencias de la alta dirección defienden la orientación al cliente; la orientación al mercado y al cliente se integra en la planificación estratégica; se desarrollan programas de marketing fuertes; se crean medidas de resultados basadas en aspectos del mercado y se fomenta el compromiso con el cliente en toda la organización. Esta propuesta es la que se formula de forma más amplia, de manera que recoge aspectos incluidos en las demás.

(Kohli y Jaworski, 1990, pág. 6) posteriormente han definido a la orientación al mercado como la generación de información por parte de toda la organización respecto a las necesidades de los clientes actuales y futuros, su diseminación a través de todos los departamentos y la respuesta de toda la organización a ese conocimiento generado y difundido.

Narver y Slater 1990 han recogido que la orientación al mercado es la cultura organizacional que de forma más efectiva y eficiente da lugar a los comportamientos necesarios para la creación de un valor superior para los compradores y como consecuencia, un resultado superior para la empresa. Han sostenido que la orientación al mercado está formada por tres componentes comportamentales: orientación al cliente; orientación a la competencia y coordinación inter $\square$ funcional (además han considerado que la empresa toma como referencia dos criterios de decisión: el largo plazo y la rentabilidad; sin embargo, estos criterios son finalmente desechados).

De esta manera, la orientación al mercado se centra en las actividades relacionadas con la adquisición y diseminación de la información sobre las necesidades de los clientes y las capacidades de los competidores (orientación al cliente y orientación a los competidores) y las actividades de coordinación de los esfuerzos de la organización para crear un producto que suponga un mayor valor para el cliente (coordinación interfuncional).

Luego, Narver, Slater y Tietje (1998) han añadido que el valor fundamental de la orientación al mercado es el compromiso de todos los miembros de la organización con la creación continua de un valor superior para el cliente. De esta forma, el principio fundamental de 
la orientación al mercado es que todos los miembros de la organización comprenden que pueden y deben contribuir de forma continua con sus habilidades y conocimientos, a la creación de un valor superior para el cliente. (p. 243)

Ruekert (1992) ha indicado que una empresa orientada al mercado obtiene información sobre sus clientes y la emplea en la formulación de estrategias que buscan la satisfacción de sus necesidades de la forma más eficaz y eficiente.

Jaworski, Kohli y Sahay (2000) han discutido sobre la existencia de dos propuestas complementarias en la orientación al mercado: "actuación en el mercado" (market driven) y "dirigiendo el mercado" (driving market). La "actuación en el mercado" se refiere a la orientación de la empresa basada en el entendimiento y la reacción a las preferencias y comportamientos de los integrantes en un mercado con una estructura determinada, mientras que la propuesta "dirigiendo el mercado" se refiere a la influencia sobre la estructura de un mercado y/o sobre el comportamiento de los integrantes de ese mercado de forma que se consiga mejorar la posición competitiva de la empresa. (Tuominen, Rajala y Möller, 2004 la han definido de la misma manera.

Se considera a la orientación al mercado como un tipo de cultura organizativa, aplicable a cualquier tipo de organización, que tiene como meta satisfacer a los clientes tanto actuales como potenciales. De una forma más operativa, la orientación al mercado debe comprender las actividades de orientación al cliente y orientación a la competencia. Consideramos, además, la necesidad de una actividad de coordinación inter $\square$ funcional que coordine las actividades anteriores en el seno de toda la organización, siendo su rol la generación de información de mercado, la diseminación de esa información y la respuesta a la misma.

Álvarez, Santos y Vásquez (2000) han mostrado que el 75\% de los estudios empíricos analizados encuentran una relación positiva entre el grado de orientación al mercado y los resultados de las organizaciones, el $17 \square$ no encuentra relación entre los esfuerzos por orientarse al mercado y los resultados de la firma y el $8 \square$ de los estudios empíricos encuentran una relación débil entre la orientación al mercado y el rendimiento de la organización.

\section{La tipología estratégica de Miles y Snow}

En el estudio de estrategia empresarial encontramos dificultades derivadas tanto de las distintas concepciones que podemos encontrar, como de los distintos niveles de análisis empleados en su formulación.

Se describen brevemente las principales clasificaciones de la estrategia a nivel de unidad de negocio para determinar cómo competir para alcanzar ventajas competitivas, con el objetivo de justificar la elección de la clasificación de Miles y Snow en esta investigación.

Porter (1980) ha diferenciado tres tipos de estrategias basadas en la forma en la que las empresas intentan conseguir y mantener una ventaja competitiva: mediante la estrategia de "liderazgo en costos"; la de "diferenciación" basada en la calidad, en el diseño, en el nombre de marca, en el servicio y creando una percepción de superioridad del producto por parte del 
consumidor y la estrategia de "especialización" con la que la empresa se concentra en un ámbito del mercado muy definido y utiliza bien la estrategia de liderazgo en costos o la de diferenciación. También identifican un cuarto tipo de estrategia con escasa rentabilidad que sitúan en una posición intermedia.

Esta tipología se centra en el ajuste entre estos tres tipos de estrategias y las fuerzas competitivas del entorno de la empresa, mientras que no se consideran ni el tipo de estructura, ni los procesos, ni los programas necesarios para implementar cada estrategia de forma efectiva.

Adicionalmente, estas categorías de Porter (1980) se han referido a acciones competitivas que la empresa realiza en la actualidad, en lugar de señalar las acciones que se proyectan para el futuro. Por el contrario, la tipología de Miles y Snow 1978 tiene como variable fundamental la planificación de la empresa respecto al cambio producto-mercado. Clasifican las unidades de negocio en cuatro tipos de estrategias: defensivas, prospectivas, analizadoras y reactivas, atendiendo a la intención o planificación del directivo y sugieren los aspectos estructurales, de proceso y de gestión que deben estar presentes en cada tipo de estrategia.

Las tipologías de Miles y Snow 1978 y Porter (1980) han representado una importante contribución para la literatura sobre estrategia empresarial. En concreto, la tipología de Miles y Snow 1978 resulta especialmente importante por su visión de la organización como sistema complejo e integrado en interacción dinámica con su entorno. Ha sido utilizada en numerosos estudios sobre estrategia empresarial y autores que la han comparado con la tipología de Porter (1980) han concluido que permite una mejor evaluación de las variables del entorno y que considera variables relacionadas con la implementación de la estrategia.

Puesto que el origen de esta tipología se basa en un análisis en profundidad de cuatro industrias diferentes, las conclusiones pueden generalizarse a distintos sectores. La principal limitación de esta clasificación es que ignora las peculiaridades industriales o del entorno ya que establece tipos de estrategias genéricas. De hecho, Miles y Snow 1978 y (Snow, Hrebiniak, 1980) han concluido que varias estrategias pueden llevar a resultados igual de buenos en cualquier industria si la estrategia está bien implementada, lo que entra en contradicción con la visión clásica de que un determinado entorno favorece la adopción de un tipo de estrategia concreta.

La tipología de Miles y Snow 1978 se ha fundamentado en tres premisas: las empresas con éxito desarrollan un proceso de adaptación a su entorno; existen cuatro orientaciones estratégicas identificables en cada industria y cualquiera de las estrategias adecuadamente implementada puede llevar a buenos resultados.

Un tema central de la literatura sobre dirección estratégica es la capacidad de adaptación al mercado de la empresa (Grant, 1991). Los estudios sobre la capacidad adaptativa tienen como supuesto básico que las empresas actúan para responder a los cambios del entorno o para crear sus propios entornos. Uno de estos estudios, basado en la definición de elección estratégica de Child (1972), se corresponde con la tipología de Miles y Snow 1978. 
Miles y Snow (1978) han propuesto que las empresas que compiten en un mercado desarrollan cuatro modelos de comportamientos estratégicos u organizativos: defensivos, prospectivos, analizadores y reactivos. La dimensión básica de esta clasificación es la capacidad de respuesta de la organización a los cambios del entorno. Identifican los distintos tipos de estrategias partiendo del ciclo adaptativo de la empresa y según la respuesta a tres cuestiones: la dimensión empresarial o estratégica, la tecnológica y la organizativa.

No se ha encontrado investigación alguna sobre la relación entre la orientación al mercado y los resultados en las pequeñas y medianas industrias ecuatorianas. Sin embargo, se ha demostrado que en estas empresas el obtener información de las necesidades de los clientes y responder rápidamente a sus expectativas son factores clave en su rendimiento. Por ende, la primera hipótesis, es la siguiente:

$\mathrm{H}_{1}$ : La orientación al mercado y los resultados tienen una relación efectiva en las nuevas pequeñas y medianas industrias ecuatorianas.

\section{Metodología}

\section{Tabla 1: Ficha técnica de la investigación}

\begin{tabular}{cc}
\hline Población objetivo: & $\begin{array}{c}\text { Empresas pequeñas y medianas del sector de “" } \\
\text { Alimentos y Bebidas” }\end{array}$ \\
Unidad Muestral & $\begin{array}{c}\text { Propietario/Director/Fundador } \\
\text { Ámbito }\end{array}$ \\
Método de recoger la información & $\begin{array}{c}\text { Provincia de Pichincha en Ecuador } \\
\text { Encuestas }\end{array}$ \\
Población $(\mathrm{N})$ & $\begin{array}{c}\text { a través de visitas físicas } \\
\text { Tamaño muestral(n) }\end{array}$ \\
Error muestral ( E) & 390 \\
Nivel de confianza(q) & 194 \\
Procedimiento de muestreo & $5 \%$ \\
Fecha de trabajo de campo & $95 \% ; 0=1.96 ; \mathrm{p}=\mathrm{q}=0.5$ \\
\hline
\end{tabular}

Nota. La muestra en relación con el total de la población representa el 49,7\%.

\section{Desarrollo de escalas de medición}

\section{Diseño del cuestionario de la encuesta}

En las encuestas se ha utilizado el método de evaluación sumaria o "escala Likert" de siete niveles, donde " 1 " significa "totalmente en desacuerdo" y "7" significa "totalmente de acuerdo".

\section{Escalas del instrumento de medición}

El instrumento diseñado para la captura de datos es el cuestionario por medio de la encuesta y se utilizó la escala de Orientación al Mercado. 
Para este estudio se considera que el enfoque cultural de la escala de (Narver y Slater, 1990) es la que se adapta mejor a esta investigación basada en los recursos y capacidades, donde la variable cultural es un recurso valioso y de difícil imitación. No se toma en cuenta el enfoque comportamental de la escala de Kohli (1993).

La encuesta de medición para la orientación al mercado es una adaptación de la escala de Narver y Slater 1990, la cual está basada en tres sub-dimensiones: i) orientación al cliente; ii) orientación a la competencia; y, iii) coordinación interfuncional.

\section{Variable de control}

\section{Dinamismo del Entorno}

Dentro de la literatura existente no se han encontrado trabajos que demuestren la correlación entre la orientación al mercado, el tamaño de la empresa, ni tampoco con el ciclo de vida (gestación, inicio y crecimiento) de las pequeñas y medianas industrias para generar resultados. Debido a esta evidencia se considera relevante la búsqueda de una variable de control diferente mediante el aporte de una investigación empírica.

De acuerdo con Kirca, Jayachandran y Bearden , las variables de control: i) dinamismo del entorno; ii) tamaño de la empresa; y, iii) estrategia genérica de la organización, impactan en el rendimiento organizacional.

En la misma línea, (María, Bojica y Fuentes, 2012) han mencionado que el dinamismo del mercado donde las organizaciones compiten, debido a los rápidos cambios en los productos, procesos, comercialización y requerimientos de los consumidores, es percibida por los directivos de las empresas, como una condición necesaria para explotar las oportunidades del mercado, a través de la formulación de estrategias apropiadas de la innovación. La ignorancia de las condiciones competitivas y de concurrencia del entorno de los negocios, impedirá que las empresas aprovechen las oportunidades de mercado, poniendo en riesgo el rendimiento y la supervivencia de la empresa.

De esta manera, las organizaciones se enfrentan con todos estos factores mencionados, que constituyen el dinamismo del entorno. Entornos tan complejos que permiten incentivar las acciones de cambio en las organizaciones y de esta manera se incrementa la eficiencia y la eficacia de la orientación al mercado y obteniendo así resultados organizacionales, ya que la empresa es hábil en conocer profundamente los requerimientos de sus consumidores y el entorno competitivo.

La capacidad de orientación al mercado se potencia en entornos hostiles, siempre y cuando la organización tenga la habilidad de responder inmediatamente a las oportunidades del mercado.

La encuesta de medición para dinamismo del entorno es una adaptación de la escala de Kohli y Jaworski (1990) y de Santos, Sanzo, Álvarez y Vásquez (2000). Estos autores han 
considerado aspectos de crecimiento del sector industrial, cambios en las preferencias del consumidor, intensidad competitiva y turbulencia tecnológica.

\section{Auto clasificación de la Estrategia}

Para este estudio se ha utilizado la auto clasificación a través del método del párrafo. El objetivo es reconocer la estrategia genérica de la empresa para el uso como variable de control.

La encuesta para la "auto clasificación a través del método del párrafo" es una adaptación de la tipología estratégica de Miles y Snow (1978).

\section{Tabla 2: Auto clasificación de la Estrategia a través del método del párrafo}

\footnotetext{
1 Nuestra empresa trata de situarse en un segmento de mercado, seguro y estable, ofreciendo una gama de productos más limitada que su competencia pero con una calidad mejor o con precios más ventajosos. No acostumbra a estar en la vanguardia de las innovaciones y deja pasar aquellas oportunidades de mercado que no guardan una relación muy directa con las actividades existentes.

2 Nuestra empresa trata de situarse en nuevos y prometedores negocios ofreciendo una gama de productos más bien limitada. A veces es pionera pero normalmente prefiere renunciar a ser la primera para estudiar con detenimiento las acciones de la competencia y así poder lanzar nuevos productos con una mejor relación calidad- precio que sus competidores.

3 Nuestra empresa opera en un amplio abanico de mercados y productos. Suele ser la primera en entrar en nuevos mercados aunque no siempre reporta una rentabilidad elevadas y actúa con rapidez para aprovechar las oportunidades de mercado.

4 Nuestra empresa no orienta su actuación hacia la elección de producto- mercado de referencia. Acostumbra a defenderse y solo reacciona en aquellos casos en se ve forzada a ellos por las presiones del entorno. Normarme es menos agresivas que sus competidores y esta menos dispuesta a asumir los mismos riesgos que adoptan estos.
}

Nota. Adaptado de la tipología estratégica de (Miles y Snow, 1978).

\section{Método estadístico para el análisis de datos}

Para verificar la hipótesis propuesta se ha empleado el modelo de ecuaciones estructurales o SEM (Structural Equation Model). Como resultado, éste modelo ha proporcionado los valores pertenecientes a la relación y más importante, un estadístico que expresa el grado en el que los datos se ajustan al modelo propuesto, confirmando su validez.

Para el análisis y el modelo de ecuaciones estructurales del estudio, se ha utilizado los siguientes programas informáticos: IBM SPSS-AMOS y Microsoft Excel.

\section{Análisis de fiabilidad}

En el estudio se incluyeron cuatro análisis:

I. Confirmar por un grupo de expertos la validez del contenido de las escalas.

II. Confirmar que no existan discrepancias en las respuestas a las encuestas.

III. Confirmar la consistencia de las escalas propuestas mediante análisis factorial.

IV. Confirmar la confiabilidad de dichas escalas. 
Las respuestas a las encuestas de los 194 propietarios de pequeñas y medianas industrias mostrarán los valores de fiabilidad de las escalas, coeficientes, correlaciones para todos los componentes de la orientación al mercado. Serán mostradas todas las correlaciones estadísticamente significativas.

A fin de cumplir los requisitos técnicos de validez y confiabilidad se han realizado las siguientes tareas:

- Se consultó a expertos y especialistas en la elaboración de los instrumentos de investigación. Y éstos confirmaron la definición de la presente investigación sobre el constructo a medir.

- El grupo de expertos valoró el nivel de relevancia que cada ítem tiene para medir el fenómeno que se pretende. Estas valoraciones permitieron seleccionar la muestra definitiva de ítems eligiendo entre los más significativos.

- Ya que el modelo propuesto tiene distintas dimensiones, el grupo de expertos revisó la asignación hecha de afirmaciones a dichas dimensiones.

- El grupo también valoró la claridad y precisión de la formulación concreta de cada ítem. Ya que la falta de claridad puede reflejar factores extraños al constructo que desea medir. Así lo han sugerido Vila, Küster y Aldás (2000).

- El grupo determinó que no se ha dejado fuera de la escala a ninguna de las dimensiones.

- Sobre la base del criterio de expertos se elaboró la versión definitiva de los cuestionarios y, por último, confirmando la validez de su contenido.

- Con los antecedentes señalados, los validadores actuaron en calidad de expertos, con los conocimientos requeridos en el proyecto, señalando cuáles son las correcciones que se deben realizar en el instrumento de investigación, para lo cual se entregaron los siguientes documentos:

- Carta de presentación, instructivo y formularios para registrar la correspondencia de cada ítem con los objetivos de la investigación, calidad técnica, representatividad y lenguaje utilizado, el instrumento y ficha del validador.

- Objetivos del instrumento, matriz de operacionalización de variables del instrumento a ser validado.

\section{Discrepancia en las respuestas a las encuestas}

En el examen del sesgo potencial de no respuesta, se ha seguido el procedimiento sugerido por Armstrong y Overtone (1977). Los resultados obtenidos permiten afirmar que la 
muestra es representativa de la población objetivo de estudio de acuerdo con el procedimiento "prueba t para muestras independientes".

\section{Análisis factorial de los componentes de las escalas}

Mediante análisis factorial se identificaron los factores que explica la configuración de la correlación de las variables observadas.

Para cada análisis factorial: matriz de correlaciones de variables, incluidos niveles de significación, determinante, inversa; matriz de correlaciones reproducida; medida de la adecuación muestral KMO (Kaiser-Meyer-Olkin) y prueba de esfericidad de Bartlett.

La consistencia de todos los componentes de todas las escalas propuestas ha mostrado un valor superior al 0.70. Mínimo admisible para el $\square$ (Nunnally, 1978. El análisis factorial exploratorio por el método de componentes principales, evalúa de manera preliminar la uni $\square$ dimensionalidad del concepto latente. Se han utilizado dos tipos de contrastes: i) Test de esfericidad de Bartlett, que en el caso de tener un nivel de significación menor a 0.05 se puede decir que la estructura factorial subyacente a los datos es aceptable; ii) KMO para la cual se ha recomendado que sea superior a 0.7 (Tres palacios, Vásquez y Bello, 2005.

\section{Tabla 3: Consistencia de los componentes de Orientación al Mercado Orientación al Cliente}

\begin{tabular}{lrr}
\hline Medida de adecuación muestral de Kaiser-Meyer-Olkin & 0.7910 \\
\hline Prueba de esfericidad de Bartlett & Chi-cuadrada & 501.918 \\
& gl & 15 \\
& Sig. & 0.000 \\
\hline
\end{tabular}

Nota. KMO Y Prueba de Bartlett para el componente Orientación al Cliente de la escala Orientación al Mercado.

\begin{tabular}{ccrrrrr}
\hline \multirow{2}{*}{ Componente } & \multicolumn{3}{c}{ Autovalores iniciales } & \multicolumn{3}{c}{$\begin{array}{c}\text { Sumatoria saturaciones al } \\
\text { cuadrado de la extracción }\end{array}$} \\
\cline { 2 - 6 } & Total & \% varianza & \% acumulado & Total & \% varianza & \% acumulado \\
\hline 1 & 3.4130 & 56.8900 & 56.8900 & 3.4130 & 56.8900 & 56.8900 \\
2 & 0.8080 & 13.4720 & 70.3620 & & & \\
3 & 0.6840 & 11.3920 & 81.7540 & & \\
4 & 0.4760 & 7.9300 & 89.6850 & & \\
5 & 0.3880 & 6.4610 & 96.1450 & & \\
6 & 0.2310 & 3.8550 & 100.0000 & & \\
\hline
\end{tabular}

Nota. Análisis de componentes principales. Varianza total explicada. Análisis factorial para el componente Orientación al Cliente de la escala Orientación al Mercado. 


\begin{tabular}{cc}
\hline & Componente \\
\cline { 2 - 2 } & 1 \\
\hline OCLI_1 & 0.790 \\
OCLI_2 & 0.780 \\
OCLI_3 & 0.790 \\
OCLI_4 & 0.710 \\
OCLI_5 & 0.770 \\
OCLI_6 & 0.800 \\
\hline
\end{tabular}

Nota. Análisis de componentes principales. (1) Componentes extraídos. Solución sin rotación.

Tabla 4: Consistencia de los componentes de Orientación al Mercado

(2) Orientación a la Competencia

\begin{tabular}{lrr}
\hline Medida de adecuación muestral de Kaiser-Meyer-Olkin & 0.8150 \\
\hline Prueba de esfericidad de Bartlett & & \\
& Chi-cuadrada & 379.199 \\
& gl & 6 \\
& Sig. & 0.000 \\
\hline
\end{tabular}

Nota. KMO Y Prueba de Bartlett para el componente Orientación a la Competencia de la escala Orientación al Mercado

\begin{tabular}{ccrrrrr}
\hline \multirow{2}{*}{ Componente } & \multicolumn{3}{c}{ Autovalores iniciales } & \multicolumn{3}{c}{$\begin{array}{c}\text { Sumatoria saturaciones al } \\
\text { cuadrado de la extracción }\end{array}$} \\
\cline { 2 - 7 } & Total & \% varianza & \% acumulado & Total & $\%$ varianza & $\%$ acumulado \\
\hline 1 & 2.8410 & 71.0370 & 71.0370 & 2.8410 & 71.0370 & 71.0370 \\
2 & 0.5190 & 12.9870 & 84.0240 & & & \\
3 & 0.3420 & 8.5560 & 92.5800 & & \\
4 & 0.2970 & 7.4200 & 100.0000 & & & \\
\hline
\end{tabular}

Nota. Análisis de componentes principales. Varianza total explicada. Análisis factorial para el componente Orientación a la Competencia de la escala Orientación al Mercado.

\begin{tabular}{cc}
\hline & Componente \\
\cline { 2 - 2 } & 1 \\
\hline OCOM_1 & 0.870 \\
OCOM_2 & 0.810 \\
OCOM_3 & 0.860 \\
OCOM_4 & 0.860 \\
\hline
\end{tabular}

Nota. Análisis de componentes principales. (1) Componentes extraídos. Solución sin rotación. 
Tabla 5

Consistencia de los componentes de Orientación al Mercado (3) Coordinación Inter-funcional

\begin{tabular}{lrr}
\hline Medida de adecuación muestral de Kaiser-Meyer-Olkin & 0.8120 \\
\hline Prueba de esfericidad de Bartlett & & \\
& Chi-cuadrada & 322.457 \\
gl & 6 \\
Sig. & 0.000 \\
\hline
\end{tabular}

Nota. KMO y Prueba de Barlett para el componente Coordinación inter-funcional. De la escala Orientación al Mercado.

\begin{tabular}{crrrrrr}
\hline \multirow{2}{*}{ Componente } & \multicolumn{3}{c}{ Autovalores iniciales } & \multicolumn{3}{c}{$\begin{array}{c}\text { Sumatoria saturaciones al } \\
\text { cuadrado de la extracción }\end{array}$} \\
\cline { 2 - 6 } & Total & \% varianza & \% acumulado & Total & \% varianza & $\%$ acumulado \\
\hline 1 & 2.7330 & 68.3190 & 68.3190 & 2.7330 & 68.3190 & 68.3190 \\
2 & 0.4970 & 80.7340 & 0.7340 & & & \\
3 & 0.2700 & 91.4010 & 91.4010 & & \\
4 & 0.3440 & 8.5990 & 100.0000 & & & \\
\hline
\end{tabular}

Nota. Análisis de componentes principales. Varianza total explicada. Análisis factorial para el componente Coordinación inter-funcional de la escala Orientación al Mercado.

\begin{tabular}{lc}
\hline & Componente \\
\cline { 2 - 2 } & 1 \\
\hline CFUN_1 & 0.810 \\
CFUN_2 & 0.860 \\
CFUN_3 & 0.850 \\
CFUN_4 & 0.860 \\
\hline
\end{tabular}

Nota. Análisis de componentes principales. (1) Componentes extraídos. Solución sin rotación.

\section{Tabla 6} Consistencia de los componentes de Resultados Organizacionales

\begin{tabular}{lrr}
\hline Medida de adecuación muestral de Kaiser-Meyer-Olkin & 0.8640 \\
\hline Prueba de esfericidad de Bartlett & & \\
& Chi-cuadrada & 803.822 \\
gl & 15 \\
Sig. & 0.000 \\
\hline
\end{tabular}

Nota. KMO y Prueba de Barlett para la escala Resultados Organizacionales. 


\begin{tabular}{crrrrrr}
\hline \multirow{2}{*}{ Componente } & \multicolumn{3}{c}{ Autovalores iniciales } & \multicolumn{3}{c}{$\begin{array}{c}\text { Sumatoria saturaciones al } \\
\text { cuadrado de la extracción }\end{array}$} \\
\cline { 2 - 7 } & Total & \% varianza & $\%$ acumulado & Total & $\%$ varianza & $\%$ acumulado \\
\hline 1 & 4.0470 & 67.4480 & 67.4480 & 4.0470 & 67.4480 & 67.4480 \\
2 & 0.8170 & 13.6160 & 81.0640 & & & \\
3 & 0.4390 & 7.3240 & 88.3880 & & \\
4 & 0.2760 & 4.6030 & 92.9910 & & \\
5 & 0.2230 & 3.7240 & 96.7150 & & & \\
6 & 0.1970 & 3.2850 & 100.0000 & & & \\
\hline
\end{tabular}

Nota. Análisis de componentes principales. Varianza total explicada. Análisis factorial para la escala Resultados Organizacionales.

\begin{tabular}{lc}
\hline & Componente \\
\cline { 2 - 2 } & 1 \\
\hline ROI_S & 0.850 \\
BFC_S & 0.910 \\
VTAS_S & 0.890 \\
R_SCLI & 0.720 \\
R_SEMP & 0.760 \\
R_GLOB & 0.910 \\
\hline
\end{tabular}

Nota. Análisis de componentes principales. (1) Componentes extraídos. Solución sin rotación.

Tabla 7

\section{Consistencia de los componentes de Dinamismo del Entorno}

\begin{tabular}{lrr}
\hline Medida de adecuación muestral de Kaiser-Meyer-Olkin & 0.7370 \\
\hline Prueba de esfericidad de Bartlett & Chi-cuadrada & 213.295 \\
& gl & 6 \\
& Sig. & 0.000 \\
\hline
\end{tabular}

Nota. KMO y Prueba de Barlett para la escala Dinamismo del Entorno.

\begin{tabular}{crrrrrr}
\hline \multirow{2}{*}{ Componente } & \multicolumn{3}{c}{ Autovalores iniciales } & \multicolumn{3}{c}{$\begin{array}{c}\text { Sumatoria saturaciones al } \\
\text { cuadrado de la extracción }\end{array}$} \\
\cline { 2 - 6 } & Total & \% varianza & \% acumulado & Total & \% varianza & $\%$ acumulado \\
\hline 1 & 2.3530 & 58.8370 & 58.8370 & 2.3530 & 58.8370 & 58.8370 \\
2 & 0.7220 & 18.0490 & 76.8860 & & & \\
3 & 0.5690 & 14.2140 & 91.1000 & & \\
4 & 0.3560 & 8.9000 & 100.0000 & & & \\
\hline
\end{tabular}

Nota. Análisis de componentes principales. Varianza total explicada. Análisis factorial para la escala Dinamismo del Entorno. 


\begin{tabular}{lc}
\hline & Componente \\
\cline { 2 - 2 } & 1 \\
\hline M_CREC & 0.750 \\
M_COMP & 0.680 \\
M_TECN & 0.840 \\
M_TMER & 0.820 \\
\hline
\end{tabular}

Nota. Análisis de componentes principales. (1) Componentes extraídos. Solución sin rotación.

\section{Fiabilidad de las escalas}

Las respuestas a las encuestas de los 194 propietarios de pequeñas y medianas industrias mostraron los valores de fiabilidad de la escala Orientación al Mercado. En base al coeficiente Alpha de Cronbach se calcularon las correlaciones de cada ítem, resultando coeficientes de relaciones positivas. Algo que se debe tomar en consideración es que el valor del $\alpha$ es el promedio de todos los coeficientes de correlación (Lind, Marchal y Wathen, 2012). Entonces, preferiblemente se debe tener una escala similar en todos los reactivos planteados.

Para la escala Orientación al Mercado, sus tres componentes: i) orientación al cliente; ii) orientación a la competencia; y, iii) coordinación inter-funcional muestran valores superiores al 0.80 de la muestra total. De acuerdo con (Nunnally, 1978) este valor es admisible en trabajos de investigación.

\section{Tabla 8}

Fiabilidad de la escala Orientación al Mercado

\begin{tabular}{|c|c|c|}
\hline \multicolumn{3}{|c|}{ Escala Orientación al Mercado (OM) } \\
\hline & Cronbach's Alpha & Item-to-total Correlation \\
\hline Orientación al cliente & 0.860 & \\
\hline Estategias de valor para clientes (OCLI1) & & 0.790 \\
\hline Objetivos satisfacción de clientes (OCLI2) & & 0.780 \\
\hline Compromiso con los clientes (OCLI3) & & 0.790 \\
\hline Comunicación frecuente con los clientes (OCLI4) & & 0.710 \\
\hline Conocimiento de necesidades de los clientes (OCLI5) & & 0.770 \\
\hline Medición de la satisfacción de los clientes (OCLI6) & & 0.800 \\
\hline Orientación a la competencia & 0.880 & \\
\hline Respuesta rápida a acciones de la competencia (OCOM1) & & 0.870 \\
\hline Equipo comercial comparte información de la competencia (OCOM2) & & 0.810 \\
\hline Ejecutivos analizamos estrategias de la competencia (OCOM3) & & 0.860 \\
\hline Objetivos en mercados con ventajas competitivas (OCOM4) & & 0.860 \\
\hline Coor dinación interfuncional & 0.861 & \\
\hline Información compartida en todas as divisiones de la empresa (CFUN1) & & 0.810 \\
\hline Coordinación de funciones en la estrategia (CFUN2) & & 0.860 \\
\hline Todas las funciones contribuyen en crear valor para los clientes (CFUN3) & & 0.850 \\
\hline Comparten recursos entres las distintas áreas de la empresa (CFUN4) & & 0.860 \\
\hline
\end{tabular}


Tabla 9

Fiabilidad de la escala Resultados Organizacionales

\begin{tabular}{|c|c|c|}
\hline \multicolumn{3}{|c|}{ Escala Resultados Organizacionales (RO) } \\
\hline & Cronbach's Alpha & Item-to-total Correlation \\
\hline Resultados or ganizacionales & 0.920 & \\
\hline Resultados ROI sujetivos (RROIS) & & 0.850 \\
\hline Resultados en beneficios (RBFC) & & 0.910 \\
\hline Resultados en ventas (RVTAS) & & 0.890 \\
\hline Resultados en satisfacción de los clientes (RSCLI) & & 0.720 \\
\hline Resultados en satisfacción de los empleados (RSEMP) & & 0.760 \\
\hline Resultados globales (RGLOB) & & 0.910 \\
\hline
\end{tabular}

Tabla 10

Fiabilidad de la escala Dinamismo del Entorno

Escala Dinamismo del Entorno (DE)

\begin{tabular}{|c|c|c|}
\hline & Cronbach's Alpha & Item-to-total Correlation \\
\hline Dinamismo del entorno & 0.770 & \\
\hline Tasa de crecimiento promedio del sector (M-CREC) & & 0.750 \\
\hline Grado de competencia en el sector industrial (M-COM) & & 0.680 \\
\hline Grado de cambio tecnológico en productos/servicios del sector (M-TECN) & & 0.840 \\
\hline Grado de cambio en las preferencias de los clientes en el mercado (M-TMER) & & 0.820 \\
\hline
\end{tabular}

\section{Validez convergente}

La validez convergente prueba que el constructo que se espera esté relacionado entre sí y la correlación entre ellos debe ser alta. Para esta validez se evaluó el constructo Orientación al Mercado que es el que posee tres componentes.

(Hair, Anderson, Tathan y Black, 1998) han recomendado que además de ser significativos, el promedio de las cargas sobre cada factor sea superior a 0.7 en promedio.

Se valida significativamente las correlaciones significativas entre los componentes de la dimensión Orientación al Mercado.

\section{Análisis factorial confirmatorio}

Dentro del análisis convergente adicionalmente se ha utilizado el análisis factorial confirmatorio (AFC). Ya formulada la hipótesis concreta sobre la relación entre indicadores y dimensiones latentes, se ha contrastado dicha hipótesis. 
Con los cuestionarios ya definidos, se ha defiido qué ítems deben medir qué dimensiones. El AFC corrige las deficiencias inherentes a la perspectiva exploratoria y conduce a una mayor concreción de la hipótesis ya que debe ser contrastada.

Adicionalmente, se ha comparado fiabilidad y validez del modelo de medida, se identificarán las relaciones causales entre dicha variable latente mediante el análisis de relaciones causales, de manera que nos permitirá conocer la influencia directa o indirecta de cada una de las variables latentes en otras y cómo están relacionadas (Byrne, 2001). En la presente investigación se ha utilizado el software IBM SPSS-AMOS para comprobar el modelo teórico mediante el modelo de ecuaciones estructurales.

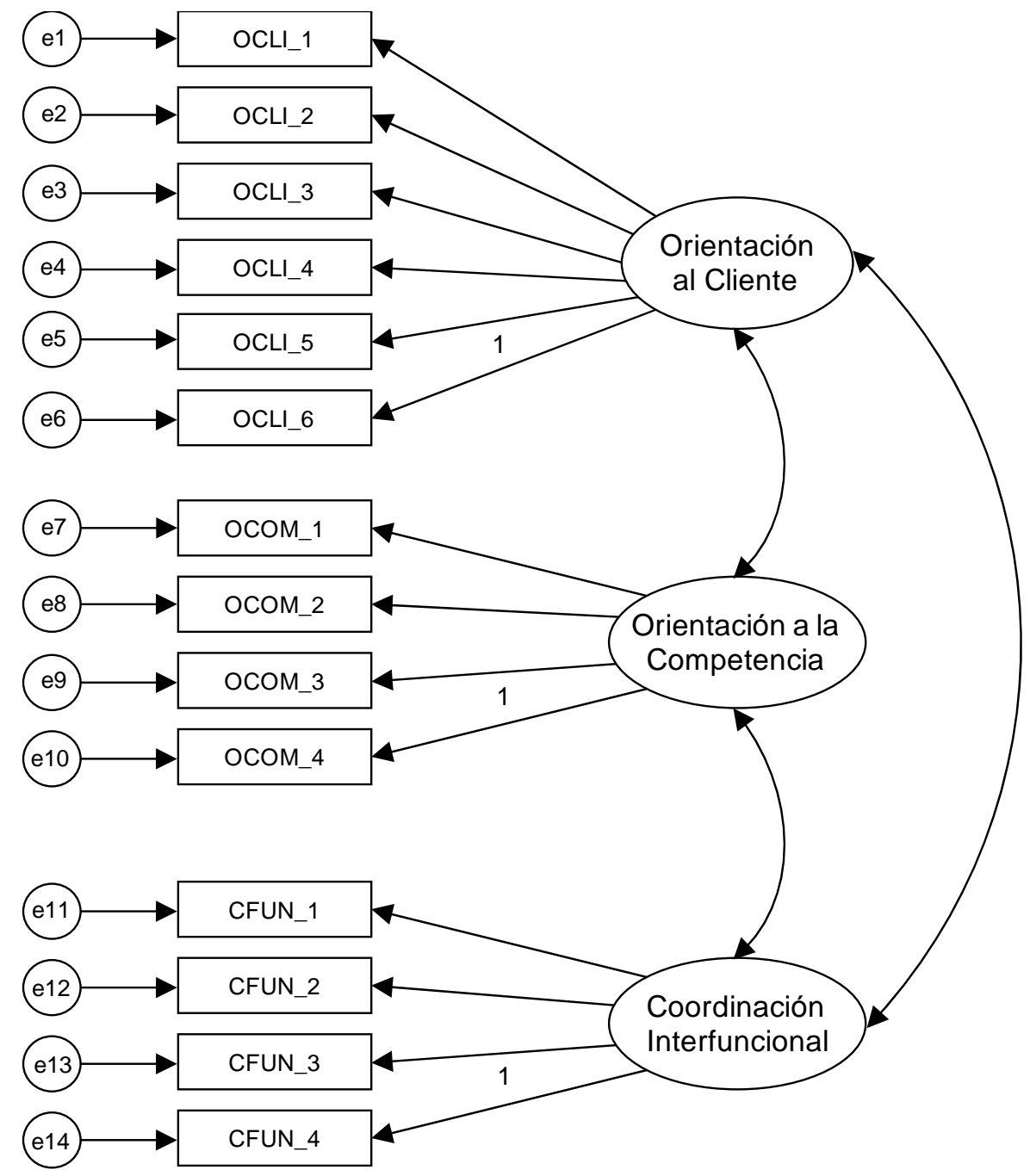

Figura 1. Análisis convergente mediante análisis factorial confirmatorio de la variable Orientación al Mercado.

\section{Validez discriminante}

La validez discriminante permite evaluar las escalas de medida de las variables latentes. De este modo la validez de contenido actúa como criterio robusto frente a determinados análisis 
estadísticos basados en covarianza. Adicionalmente, son las más utilizadas en las ciencias sociales y que se han ligado más estrechamente a la idea de validez del constructo.

(Campbell y Fiske, 1959) han afirmado que para que unas medidas sean válidas, las de un mismo constructo deben correlacionar altamente entre ellas (convergente) y que dicha correlación debe ser mayor que la que exista con respecto a las medidas propuestas para otro constructo distinto (discriminante).

El análisis muestra correlación estadísticamente significativa de los ítems de cada elemento para con el factor de su componente.

\section{Resultados y análisis}

\section{Resultado del modelo con ecuaciones estructurales}

El análisis de los resultados ya que se ha demostrado que la escala orientación al mercado y los resultados organizacionales, logra relación y son válidas.

Sí existe una relación efectiva entre la orientación al mercado y los resultados organizacionales en las pequeñas y medianas empresas ecuatorianas, es estadísticamente significativa: 0.731. Las investigaciones por parte de (Jaworski y Kholi, 1993); (Narver y Slater, 1990) y (Pelham, 1999) han presentado resultados similares en comparación con este estudio empírico.

La variable de control "Dinamismo del Entorno" no tiene una mayor influencia significativa en la relación orientación al mercado y los resultados organizacionales. Es decir, su grado de influencia es baja en el presente estudio.

\begin{tabular}{ccc}
\hline Bajo & Alto & Prueba t \\
\hline 0.562 & 0.734 & 5.75 \\
\hline
\end{tabular}

\section{Discusión y conclusiones}

Con la evidencia empírica se confirma que la presente investigación coincide con los estudios realizados por (Kohli, Jaworski, 1990) y (Narver y Slater, 1990) donde la orientación al mercado permite a las organizaciones adaptarse a las necesidades y requerimientos del mercado.

Como contribución del presente estudio, éste tiene un impacto social que se centra principalmente en los emprendedores que tienen la idea de negocio y quienes serán los futuros inversionistas, generadores de empleo y producción. Al tener identificados los principales factores de éxito se podrá dar la pauta necesaria para que los futuros emprendimientos puedan tener mejores oportunidades de permanencia en el mercado y aumenten su tiempo de vida.

Se ha demostrado que sí existe una relación efectiva entre la orientación al mercado y los resultados organizacionales en las empresas ecuatorianas, de acuerdo a las definiciones más 
influyentes de los autores: Kohli y Jaworski (1990) y las de Narver y Slater (1990), este estudio empírico coincide también con su estudio. Esto es, cuando las empresas recogen la información de las necesidades de los clientes actuales y futuros (sub-dimensión: orientación al cliente); la comunicación hacia todos los colaboradores de la organización, llamada sub-dimensión "coordinación inter funcional"; analizar y discutir las estrategias de los competidores (sub-dimensión: orientación a la competencia) no para imitarlas, sino para diseñar estrategias diferentes, estas sub-dimensiones que conforman la orientación al mercado en su conjunto, permiten tener una cultura organizacional que crea un valor superior para los clientes o consumidores, como consecuencia, un mejor resultado en los beneficios empresariales.

Esta correlación tiene un mayor impacto cuando la empresa toma en cuenta al entorno externo, esto significa que debe estar atenta al crecimiento del sector, al grado de competencia, al cambio tecnológico en los productos/servicios que se ofertan y al grado de cambio en las preferencias de los clientes. Este estudio empírico coincide con los resultados de Kohli y Jaworski (1990) donde han demostrado que no solamente se requiere conocer sobre el cliente, sino que también que todo esto requiere años para que la organización desarrolle productos/servicios de valor para el consumidor.

En el estudio realizado sobre la orientación al mercado, todavía se puede observar que las pequeñas y medianas industrias ecuatorianas de este sector productivo, poseen un tipo de cultura organizacional que tiene como meta satisfacer a los clientes actuales y potenciales. Pero, estas empresas todavía no comprenden del todo las actividades de coordinación inter funcional y orientación a la competencia. De acuerdo a esta investigación, se sugiere que las direcciones de las empresas desarrollen su actividad de comunicación interna y también implementen estrategias diferenciadoras, ya sea creando y promocionando una categoría diferente para productos genéricos o incrementando el grado de diferenciación en sus productos/servicios.

Se muestra que alrededor del 15\% de la muestra (30 empresas) son organizaciones de tipo reactivas y el 33\% (64 empresas) son de tipo defensivas. Si juntamos a estas 94 empresas, tenemos un $48 \%$ de organizaciones de la muestra que sí toman en cuenta el dinamismo del entorno, pero en su mayoría de manera tardía, esto hace que el impacto en los resultados empresariales baje o no sea superior al promedio en el sector estudiado.

¿Qué significa ser defensiva o reactiva? Las organizaciones de la muestra analizada, son 94 empresas con estrategia defensiva que se enfocan en mercados pequeños, se vuelven especialistas para su grupo objetivo. Se centran en mejorar sus operaciones en cuanto a producción y control de costos. Pero su debilidad (15\% de la muestra) se encuentra en que no responden de forma efectiva a los cambios e incertidumbres; carecen de una relación estrategia-estructura y no realizan ajustes hasta que no son forzadas por las presiones del entorno.

López y Sánchez (2007) han demostrado que existe una relación entre la orientación al mercado y los resultados empresariales y se refuerza cuando se adopta una estrategia defensiva. El presente estudio empírico también coincide con estos dos autores, es decir, las empresas ecuatorianas mejoran sus resultados al tener una estrategia defensiva. 
El 52\% de las 194 empresas que pertenecen a la muestra estudiada, son de tipo prospectivas (31\%) y de tipo analizadoras (21\%). Las prospectivas (60 empresas de la muestra), se centran más en diversificar su línea de productos, enfatizan en innovación, están orientadas al cliente ofreciendo productos nuevos en el mercado, pero dicho énfasis en la orientación innovadora les impide ser eficientes. La razón es que requieren tener una mayor orientación al mercado.

Las analizadoras (40 empresas), mantienen un dominio producto-mercado estable; observan las ideas de los competidores y luego, adoptan las más prometedoras; su portafolio de productos es básico y limitado; poseen énfasis en la producción, los procesos y el marketing. De la misma manera, se sugiere que deben desarrollar una mayor orientación al mercado en la sub-dimensión de orientación a la competencia, el objetivo es observar y analizar las estrategias de ellos y mejorarlas, es decir, tener un mayor grado de diferenciación y no copiar las ideas más prometedoras de la competencia. De esta manera, aumenta el impacto en los resultados deseados.

Los hallazgos del presente trabajo de investigación tienen relevancia en los fundadores, directivos, gerentes y responsables de la gestión de las empresas, ya que impactan directamente en los beneficios organizacionales. Como se ha explicado en esta investigación, en el Ecuador existen cada año una mayor cantidad de creación de negocios y los mencionados estudios en su ranking de emprendimientos en América del Sur posicionan al país en el puesto No. 1, pero también está ubicado en el ranking de negocios cerrados en el puesto No. 8.

De acuerdo a las conversaciones con los directores/fundadores de las empresas, se puede observar que la cultura organizacional de estas empresas ecuatorianas es la consecución de resultados en el corto o máximo en el mediano plazo, un paradigma que se debe romper. Hay mucho trabajo todavía por hacer, esperamos que la presente investigación y sus hallazgos impacten de manera positiva sobre este pensamiento. Anteriormente, en este trabajo se ha mencionado que el punto inicial de la orientación al marketing es la información del mercado, entendido como algo más que las necesidades y preferencias de los clientes, ya que también incluyen el control de las acciones de la competencia y sus efectos sobre los clientes, así como el análisis de los factores externos como la regulación legal, la tecnología o el entorno. Esto sugiere que las organizaciones se adelanten a las necesidades del cliente, siendo conscientes de que se necesitan años para desarrollar los productos que los satisfagan.

\section{Bibliografía}

Álvarez, L., Santos, M., Vázquez, R. (2000). Análisis cultural y operativo de la orientación al mercado. Efectos moderadores en la relación OM-Resultados. Revista Española de Investigación en Marketing ESIC, Vol. 4, No. 1, pp. 7-41.

Campbell, D.T., Fiske, D.W. (1959). Convergent and discriminant validation by the multitraitmultimethod matrix. Psychol. Bull, Vol. 56(2), pp. 81-105.

Day, G.S. (1994a). The capabilities of market-driven organizations. Journal of Marketing, Vol. 58 , pp. 37-52. 
Day, G.S. (1994b). Continuous learning about markets. California Management Review, Vol. 36, No. 4, pp. 9-31.

Deshpandé, R., Farley, J.U., Webster, F.E. Jr. (1993). Corporate culture, customer orientation, and innovativeness in Japanese firms: a quadrad analysis. Journal of Marketing, Vol. 57, No. january, pp. 23-37.

Deshpandé, R., Farley, J.U. (1998). Measuring market orientation generalization and synthesis. Journal of Market Focused Management, Vol. 2, No. 3, pp. 213-232.

Esteban, A., Campos, M., Molina C. (2002). Market orientation in service: a review and analysis. European Journal of Marketing, Vol. 36, No. 9-10, pp. 1003-1021.

Gallo, J.G. (2017). Impacto de la orientación al mercado y el desarrollo de capacidades empresariales en los resultados de las pequeñas y medianas industrias ecuatorianas. (Tesis doctoral sin publicar). Facultad de Ciencias Económicas. Universidad Nacional de Córdoba.

Greenley, G. (1995). Market orientation and company performance: empirical evidence from UK companies. British Journal of Management, Vol. 6 No. December, pp. 1-13.

Hair, J.F. Jr., Anderson, R.E., Tatham, R.L., Black, W.C. (1998). Multivariate Data Analysis (5 ed.), Upper Saddle River, NJ: Prentice Hall.

Jaworski, B.J., Kohli, A.K. (1993). Market orientation: antecedents and consequences. Journal of Marketing, Vol. 57, No. 3, pp. 53-70.

Kennedy, K.N., Goolsby, J.R., Arnould, E.J. (2003). Implementing a customer orientation: extension of theory and application. Journal of Marketing, Vol. 67, October, pp. 67-81.

Kirca, A., Jayachandran, S., Bearden, W. (2005). Market orientation: a Meta analytic 224 review and assessment of its antecedents and impact on performance. Journal of Marketing, Vol. 69 , pp. 24-41.

Kohli, A.K., Jaworski, B.J. (1990). Market orientation: the construct, research propositions and managerial implications. Journal of Marketing, Vol. 54, No. 2, pp. 1-18.

Kohli, A.K., Jaworski, B.J., Kumar, A. (1993). MARKOR a measure of market orientation. Journal of Marketing Research, Vol. 30, No. 4, pp. 467-477.

Kotler, P., Cámara, D., Grande, I., Cruz, I. (2000). Dirección de Marketing. Edición del Milenio, Madrid, España: Pretince Hall Iberia.

Levitt, T. (1983). After the sale is over... Harvard Business Review, Vol 61, No. 5, pp. 87-93. 
Lind, A., Marchal, G., Wathen, S. (2012). Statistical Technics in Business \& Economics. México: McGraw-Hill.

Llonch, A.J. (1993). Orientación al mercado y competitividad de la empresa. (Barcelona: EADA Gestión).

Llonch, A.J., Waliño, S., (1996). Efectos de la orientación al mercado en los resultados de la empresa. Revista Española de Investigación de Marketing ESIC, No. enero-marzo, pp. 939.

María, D.M., Bojica, A.M., Fuentes, F. (2012). Knowledge acquisition and corporate entrepreneurship: insights from Spanish SMEs in the ICT sector. Journal of World Business, Vol.47, No. 3, pp. 397-400.

McNeal, J.U., Lamb, C.W. (1980). Marketing orientation in nonprofit sector: the case of hospital. Journal of the Academy of Marketing Science, Vol. 8, No. 1, pp. 26-32.

Miles, M.P., Arnold, D.R. (1991). The relationship between marketing orientation and entrepreneurial orientation. Entrepreneurship: theory and practice, pp. 49-59.

Miles, R.E., Snow, C.C. (1978). Organizational strategy structure and process. New York: USA: McGraw-Hill.

Miles, R.E., Snow, C.C. (1978). Organizational strategy structure and process. McGraw-Hill.

Citado en Rodríguez, J.M. (2001). Configuraciones estratégicas y sistema de gestión de recursos humanos: una revisión de la tipología de Miles y Snow. Documento de trabajo, No. 237/01. Universidad de Oviedo.

Miles, R.E., Snow, C.C., Meyer, A.D., Coleman, H.J. (1978). Organizational strategy, structure and process. Academy of Management Review, Vol. 3, No. 3, pp. 546-562.

Narver, J.C., Slater, S.F. (1990). The effect of a market orientation on business profitability. Journal of Marketing, Vol. 54, No. 4, pp. 20-35.

Narver, J.C., Slater, S.F., Tietje, B. (1998). Creating a market orientation. Journal of Market Focused Management, Vol. 2, pp. 233-236.

Nunnally, J.C. (1978). Psychometric theory. New York: McGraw-Hill, N.Y.

Pelham, A.M. (1997). Mediating influences on the relationship between orientation and profitability in small industrial firms. Journal of Marketing Theory and Practice, Vol. 5, No. 3, pp. 1-23.

Pelham, A.M. (1999). Influence of environment, strategy, and market orientation on performance in small manufacturing firms. Journal of Business Research, Vol. 45, No. 1, pp. 33-46. 
Porter, M.E. (1980). Competitive strategy, New York, USA: Free Press.

Porter, M.E. (2006). Estrategia y ventaja competitiva. México D.F., México: Deusto.

Ruekert, R.W. (1992). Developing a market orientation: an organizational strategy perspective. International Journal of Research in Marketing, Vol. 9, pp. 225-245.

Ruiz, M.A., Pardo, A., San Martín, R. (2010). Modelos de Ecuaciones Estructurales. Papeles del Psicólogo, Vol. 31, No. 1, pp. 34-45.

Santos, M.L., Sanzo, M.J., Álvarez, L.I., Vázquez, R. (2002a). El aprendizaje organizativo y la orientación al mercado como recursos empresariales: interacciones y efectos sobre la competitividad. Revista Española de Investigación de Marketing ESIC. Vol. 6, No. 1, pp. $7-36$.

Shapiro, B. (1988). What the hell is market-oriented? Harvard Business Review, Vol. novemberdecember, pp. 119-125.

Tuominen, M., Rajala, A., Möller, K. (2004). Market-driving versus market-driven: divergent roles of market orientation in business relationships. Industrial Marketing Management. Vol. 33, No. 3, pp. 207-217.

Trespalacios, J., Vázquez, R., Bello, L. (2005). Investigación de mercados: métodos de recogida y análisis de la información para la toma de decisiones de marketing. España. Ediciones Paraninfo.

Vila, N., Küster, I., Aldás, J. (2000). Desarrollo y validación de escalas de medida en marketing. Universitat de València, p. 64. Recuperado de http://www.uv.es/aldas/resources/Docencia/URV/3.Apuntes_ESC.pdf

Walker, O.C., Ruekert, R.W. (1987). Marketing's role in the implementation of business strategies: A critical review and conceptual framework. Journal of Marketing, Vol. 51, No. 3, pp. 15-33.

Webster, F.E. Jr. (1988). Rediscovering the marketing concept. Business Horizons, Vol. 31, No. May-June, pp. 29-39.

Weston, R., Gore, P.A. (2006). A brief guide to structural equation modeling. The Counseling Psychologist, Vol. 34(5), pp. 719-751.

Wrenn, B. (1997). The market orientation construct: measurement and scaling issues. Journal of Marketing Theory and Practice. Vol. 5, No. 3, pp. 31-54. 